\title{
Thérapie de groupe EMDR pour des femmes agressées sexuellement au Congo
}

\author{
Michel Allon \\ Hoshaya, Isrä̈l
}

\begin{abstract}
Chaque année, des milliers de femmes sont victimes d'agressions sexuelles en République démocratique du Congo. En 2008, I'auteur a été invité en République démocratique du Congo afin d'aider au développement et à l'évaluation d'une approche thérapeutique brève pouvant traiter efficacement des femmes victimes d'agression sexuelle et souffrant de stress post-traumatique. Dans cette étude de terrain, 37 femmes ont reçu, soit 2 séances de thérapie individuelle $(n=8)$ utilisant la désensibilisation et le retraitement par les mouvements oculaires (EMDR), soit 2 séances de thérapie de groupe $(n=29)$ utilisant le protocole EMDR intégratif de traitement de groupe (EMDR-PITG). Les résultats de l'étude ont montré une amélioration importante des notes à l'échelle d'unités subjectives de perturbation (SUD). Les notes SUD des femmes ayant suivi des séances de thérapie individuelle ont indiqué une diminution significativement plus forte que celles des participantes traitées en thérapie de groupe. Avant le traitement, toutes les femmes de l'étude avaient rempli l'échelle d'impact de l'événement (IES - Impact of Event Scale), avec des notes bien au-dessus du seuil indiquant un état de stress post-traumatique. Ces mesures avaient également été relevées, deux semaines après la fin de leur traitement, auprès des femmes $(n=6)$ du premier des quatre groupes EMDR-PITG, avec une diminution significative des notes. De plus, beaucoup de femmes ont spontanément rapporté l'élimination de douleurs corporelles après la thérapie. Cette étude suggère l'importance de mener davantage de recherches et de développement.
\end{abstract}

Mots-clés: République Démocratique du Congo ; désensibilisation et retraitement par les mouvements oculaires (EMDR) ; protocole EMDR intégratif de traitement en groupe (EMDR-PITG) ; résultat du traitement ; étude de terrain ; rescapées d'agressions sexuelles

$\mathbf{E}$ n 2008, l'auteur a été invité à accompagner une délégation médicale de quatre médecins gynécologues en République démocratique du Congo afin d'aider, dans la province du Sud-Kivu, au traitement de femmes qui avaient été victimes d'agressions sexuelles au cours de la guerre civile. La mission était organisée par l'organisation non gouvernementale (ONG) d'assistance humanitaire israélienne Latet, en partenariat avec l'ONG Malteser International, qui opérait à l'est du Congo, apportant de l'aide aux femmes victimes d'agressions.

Sur une période de 36 mois, s'étendant entre janvier 2005 et décembre 2007, Malteser International avait dénombré environ 20500 femmes congolaises victimes de viol dans la province du Sud-Kivu. Étant donné les nombreux obstacles empêchant les victimes de viol de rendre compte de leurs agressions, le nombre réel de violences sexuelles était probablement beaucoup plus élevé (Steiner et coll., 2009). Malteser International, outre une assistance médicale et économique, tentait également de fournir aux femmes une assistance psychologique. Ils utilisaient « l'écoute active » en séances de counseling, technique qui reste la technique thérapeutique recommandée dans les documents gouvernementaux congolais officiels en coordination avec les Nations Unies (Ministère de la santé publique, 2012). Malteser International a réalisé que ces techniques thérapeutiques ne permettaient

This article originally appeared as Allon, M. (2015). EMDR Group Therapy With Women Who Were Sexually Assaulted in the Congo. Journal of EMDR Practice and Research, 9(1), 28-34. Translated by François Mousnier-Lompré. 
pas de répondre aux besoins de ces femmes et demanda donc à Latet d'adjoindre à la mission médicale un psychologue formé dans le traitement des symptômes post-traumatiques.

Les personnes travaillant en santé mentale en République démocratique du Congo sont peu nombreuses. Le nombre de psychologues pour 100000 personnes est de 0,015 , et le nombre de travailleurs sociaux de 0,018 (Organisation mondiale de la santé, 2011). Étant donné le nombre très élevé de femmes nécessitant un traitement et les ressources de santé mentale limitées, l'auteur décida d'évaluer le modèle de groupe de la thérapie de désensibilisation et de retraitement par les mouvements oculaires (EMDR) ainsi que le protocole EMDR intégratif de traitement en groupe (EMDR-PITG), afin de déterminer son efficacité à traiter certaines de ces femmes, et dans la mesure où cela s'avérerait un moyen thérapeutique plus efficient et moins coûteux.

\section{La désensibilisation et le retraitement par les mouvements oculaires}

L’EMDR a été développée par Francine Shapiro dans le but de traiter les personnes souffrant d'état de stress post-traumatique (ESPT) et la première publication de ses recherches remonte à 1989 (Shapiro, 1989). Ce modèle postule que la base de la souffrance psychologique trouve son origine dans les émotions et les sensations physiques liées à des événements traumatiques non résolus et dans un stockage inadéquat de ces souvenirs dans le système mnésique (Van Rood $\&$ De Roos, 2009). L'EMDR consiste à faire remémorer au patient les souvenirs spécifiques de l'événement traumatique pendant que le thérapeute, ou le patient lui-même, exercent des stimulations bilatérales : soit des mouvements oculaires horizontaux, soit une alternance gauche-droite de tapping sur une partie du corps du client. Des méta-analyses ont établi l'efficacité de l'EMDR dans le traitement de l'ESPT (Bisson $\&$ Andrew, 2009). Dans leur livre, Shapiro et Forrest (1997) ont montré l'efficacité de l'EMDR pour traiter les ESPT de femmes ayant été victimes de viol. Des études cliniques ont démontré l'efficacité de l'EMDR dans le traitement de femmes victimes de viol (Rothbaum, 1997 ; Rothbaum, Astin \& Marsteller, 2005).

Lorsqu'un désastre à grande échelle survient, par exemple un tremblement de terre, il est parfois difficile de traiter les victimes souffrant d'ESPT sur une base individuelle à cause du nombre élevé des victimes et du manque éventuel de professionnels formés. En 1997, l'EMDR-PITG a été développé à la suite de l'ouragan Pauline au Mexique (Jarero,
Artigas \& Hartung, 2006). Les participants dessinent le trauma qu'ils ont vécu et notent l'émotion résiduelle liée à ce trauma, l'évaluant à l'aide de l'échelle d'unités subjectives de perturbation (SUD) (où $0=$ aucune perturbation et $10=$ la pire perturbation possible). Tout en se concentrant sur leur dessin, les participants appliquent sur eux-mêmes la stimulation bilatérale grâce à une technique appelée le papillon (Artigas, Jarero, Mauer, Lopez Cano \& Alcala, 2000), où ils croisent les bras sur leur poitrine et tapotent alternativement leurs épaules. Ce processus est répété avec une série de dessins, chaque dessin montrant généralement moins de perturbation que le précédent. Les participants traitent ainsi leurs souvenirs traumatiques. Des rapports publiés rapportent le succès rencontré par l'EMDR en thérapie de groupe avec des enfants et des adultes (Jarero, \& Artigas, 2010 ; Jarero, Artigas \& Montero, 2008). L'auteur avait déjà utilisé l'EMDR-PITG avec succès sur des adultes en Israël, en 2006, lors de la seconde guerre du Liban.

\section{Méthode}

L'auteur s'est rendu dans deux villes de l'est du Congo, Kakwende et Kasika, accompagné de trois assistants : deux psychologues congolais formés sur place et une infirmière psychiatrique congolaise.

Les participantes et leur assignation à une forme de traitement

Les 37 femmes ayant participé à l'étude avaient été sélectionnées par les coordinateurs locaux du programme d'assistance formés par Maltuser International. Chacune se rendait au site de traitement le plus proche de chez elle. Aucun critère formel d'acceptation n'était requis mais toutes les femmes avaient été victimes d'agressions sexuelles. Les coordinateurs de Maltuser ont décidé ensuite, et ce uniquement d'après leur jugement subjectif, si les femmes sélectionnées pouvaient participer à la thérapie de groupe ou si elles avaient besoin de séances de thérapie individuelle. Huit femmes ont été assignées à des séances de thérapie individuelle et vingt-neuf autres à l'un ou l'autre de quatre groupes de thérapie, qui prenaient chacun en charge 6 à 8 femmes. À la suite du traitement en groupe, six femmes issues de la thérapie de groupe et dont les notes à l'échelle SUD restaient élevées ( 6 ou plus) ont reçu une séance EMDR individuelle additionnelle.

\section{Mesures}

L'échelle d'impact des événements. L'échelle d'impact des événements (IES - Impact of Events Scale) est un moyen de mesure répandu du stress 
post-traumatique (Horowitz, Wilner \& Alvarez, 1979). Les 15 items du questionnaire IES sont évalués sur une échelle de fréquence en 4 points (où $0=$ pas du tout, $1=$ rarement, $3=$ parfois, $5=$ souvent). L'IES produit un résultat total variant entre 0 et 75 . Nous avons utilisé dans cette étude le seuil diagnostique recommandé par Neal et coll. (1994) qui ont déterminé sur une base empirique qu'un résultat IES de 35 permettait d'identifier avec fiabilité un ESPT chez $89 \%$ des personnes évaluées. Ce seuil clinique possède une haute sensibilité et spécificité (Brewin, 2005). Les items ont été traduits $\mathrm{du}$ français en swahili, langue prédominante dans cette partie du pays, par les trois assistants qui parlaient couramment les deux langues. L'IES a été employée oralement par l'un des trois assistants, car beaucoup des femmes interrogées n'avaient que peu ou pas été scolarisées et ne savaient pas lire. Nous avons effectué ces mesures chez toutes les femmes lors du prétraitement. Les femmes $(n=6)$ du premier des quatre groupes d'EMDR-PITG ont également fait le test à nouveau en post-traitement, 2 semaines après la fin du traitement de groupe.

L'échelled'unités subjectivesdeperturbation. L'échelle SUD développée par Wolpe (1969) est utilisée pour mesurer l'intensité subjective de la perturbation ou de la souffrance ressentie par un individu. L'intensité de la perturbation est évaluée sur une échelle de 0 à 10 (où $0=$ aucune perturbation et $10=$ une perturbation très importante). Des recherches menées par Kim, Bae \& Park (2008) ont confirmé la validité des mesures SUD et son efficacité à refléter l'intensité des symptômes d'un patient avant la thérapie EMDR.

\section{Traitement}

Les femmes sélectionnées pour la thérapie individuelle ont reçu deux séances de thérapie EMDR standard. Les femmes sélectionnées pour la thérapie de groupe ont reçu deux séances de la procédure EMDRPITG (Jarero et coll., 2006) avec quelques modifications (voir appendice). Les adaptations apportées au protocole d'origine sont les suivantes :

1. Certaines femmes ne savaient pas dessiner ou n'avaient jamais tenu un crayon auparavant. Pour ces cas-là, les assistants écrivaient sur une feuille ce que les participantes leur décrivaient avant et pendant le traitement.

2. Certaines de ces femmes n'avaient aucune connaissance des nombres et nous avons donc substitué des mots descriptifs aux nombres pour les mesures des niveaux de SUD (aucune perturbation, très peu de perturbation, un peu de perturbation, etc.)
3. La phase de scan corporel (phase 6) a été éliminée parce que nous nous sommes rendu compte que les femmes de l'étude ne pouvaient pas localiser dans quelle partie du corps elles ressentaient leurs émotions.

Nous avons utilisé comme équipement des feuilles de papier A4 et des marqueurs de couleur.

\section{Résultats}

Sur les 29 femmes traitées avec deux séances de thérapie de groupe, 6 nécessitaient et ont accepté de recevoir une séance individuelle additionnelle, à la suite de la thérapie de groupe, afin de compléter le retraitement des conséquences psychologiques des agressions sexuelles dont elles avaient été victimes, parce que leur résultat sur l'échelle SUD, à la fin de la thérapie de groupe, était au-dessus de 6. Cependant, deux autres femmes, dont les notes à l'échelle SUD étaient supérieures à 6 à la fin de la thérapie de groupe, refusèrent de poursuivre leur traitement en thérapie individuelle. Une autre femme présentant un résultat SUD élevé à la fin de la thérapie de groupe n’a pas été reçue en séance individuelle en raison de problèmes médicaux qui ont nécessité son hospitalisation.

\section{Prétraitement}

En prétraitement, les notes IES de toutes les femmes allaient de 30 à 70 avec une moyenne à 51,1 $(\sigma=9.3)$. La moyenne des notes IES de toutes les femmes adressées en thérapie de groupe était de 50,1 $(\sigma=9.3)$. La moyenne des notes IES des femmes orientées vers la thérapie individuelle était de $54,9(\sigma=8.8)$. Il n'y avait pas de différence significative $(t(35)=0.84, p>0.20)$ entre les moyennes des notes IES pour les deux groupes de femmes. Dans cette étude, nous avons employé le seuil diagnostique de 35. Ce seuil a été développé empiriquement et est recommandé par Neal et coll. (1994). Deux des femmes assignées en thérapie de groupe présentaient un résultat IES inférieur à 35 , mais supérieur à 30 . Les moyennes des notes IES en prétraitement étaient donc bien supérieures au seuil clinique, suggérant une forte probabilité que la plupart des femmes de l'étude souffraient effectivement d'ESPT. La moyenne des notes de l'évaluation SUD de toutes les femmes participant à l'étude, au début de la thérapie, était de $9.0(\sigma=1.2)$.

\section{Post-traitement}

Notes des unités subjectives de perturbation. L'évaluation moyenne à l'échelle SUD pour toutes les femmes à la fin de la thérapie était $4.3(\sigma=3.0)$. 
TABLEAU 1. Participantes de thérapie de groupe ayant une note SUD $>6$ à la fin de la deuxième séance

\begin{tabular}{cccc}
\hline Participante & Note SUD en prétraitement & Note SUD post-traitement & Note SUD post-traitement individuel \\
\hline 1. & 9 & 6 & 2 \\
2. & 9 & 6 & 8 \\
3. & 10 & 8 & 8 \\
4. & 10 & 6 & 2 \\
5. & 10 & 8 & 3 \\
6. & 10 & 6 & 1 \\
\hline
\end{tabular}

Note $:$ SUD $=$ unités subjectives de perturbation

Cette amélioration était statistiquement significative $(t(36)=9.83, p<0.0001)$. Les femmes de la thérapie de groupe rapportèrent une diminution importante des notes SUD, avec une moyenne allant de $9.0(\sigma=$ $1.3)$ au début de la thérapie, à $4.8(\sigma=2.9)$ à la fin du traitement, $t(28)=8.29, p<0.0001$. Ces notes SUD de thérapie de groupe en post-traitement incluent les évaluations des 29 femmes après la fin de la thérapie de groupe ; elles n'incluent pas la baisse des notes SUD des 6 femmes qui ont reçu une séance additionnelle de thérapie individuelle. La note SUD moyenne de ces six femmes après leur thérapie individuelle était de $2.8(\sigma=2.6$; voir tableau 1$)$

Ainsi que nous l'avons déjà dit, neuf femmes issues de la thérapie de groupe avaient des notes SUD finales supérieures à 6 . La moyenne des notes SUD à la fin des deux séances pour les 20 autres femmes en thérapie de groupe était 3.6, avec neuf femmes ayant obtenu une note SUD finale de 3 ou moins.

Les femmes en thérapie individuelle $(\mathrm{n}=8)$ rapportèrent une diminution significative de la moyenne des notes SUD, allant de $9.3(\sigma=0.9)$ à $1.9(\sigma=2.2)$ à la fin de la thérapie $(t(7)=10.85, p<0.0001)$. Nous avons observé une différence significative dans les notes SUD finales entre les femmes ayant reçu des séances de thérapie individuelle et celles qui avaient reçu des séances de thérapie de groupe $(t(35)=2.63$, $p<0.01)$.

Résultats de l'échelle d'impact des événements. Faute de temps, l'évaluation IES a été passée en post-traitement seulement par les femmes $(n=6)$ du premier groupe de thérapie deux semaines après la fin de leurs deux séances de thérapie de groupe. Alors que la moyenne des notes IES de ce groupe en prétraitement était de 52, elle était passée à 33 deux semaines après la fin de la thérapie. Cette diminution des notes IES est significative sur le plan statistique $(t(5)=2.50$, $p<0.03)$.

\section{Discussion}

Cette étude de terrain visait à déterminer si la thérapie de groupe EMDR et l'utilisation de l'EMDRPITG (Jarero et coll., 2006) pouvaient être des outils efficaces et efficients dans le traitement de femmes adultes victimes d'agressions sexuelles en République démocratique du Congo. L'étude a démontré que deux séances de thérapie individuelle EMDR standard produisaient de meilleures évaluations SUD que deux séances de thérapie EMDR de groupe. Cela ne signifie pas que l'EMDR-PITG n'est pas efficace dans le traitement de l'ESPT, mais qu'il faut prendre en compte les différents facteurs lorsqu'on fait de la thérapie de groupe.

\section{Efficience et efficacité du traitement individuel}

Le traitement EMDR individuel s'est montré efficace. Deux séances de thérapie EMDR individuelle ont été appliquées à huit femmes dont la moyenne des notes IES en prétraitement était de 54.9 et celle des notes au SUD était de 9.3. Après seulement deux séances EMDR standard, la moyenne des notes de l'échelle SUD avait chuté à 1.9. Comment expliquer une telle diminution en seulement deux séances ? Je l'ignore. Est-ce l'impact du Docteur blanc venu de loin pour prodiguer des soins à ces femmes ? Toutefois, la rapidité et l'efficacité de ces résultats cadrent bien avec ceux rapportés par Jarero et ses collègues (Jarero, Artigas, Uribe \& Miranda, 2014). La thérapie EMDR a eu sur ces femmes congolaises les résultats les plus rapides et les plus efficaces qu'il m’ait été donné de voir, et ce phénomène mériterait de faire l'objet d'un examen plus approfondi.

Une autre preuve de l'efficacité de la thérapie a été la disparition des symptômes psychosomatiques de ces femmes. Beaucoup d'entre elles, en thérapie individuelle ou en thérapie de groupe, disaient souffrir, 
avant la thérapie, de douleurs quotidiennes au basventre ou dans la région lombaire et ont rapporté, à la fin du traitement, la disparition complète de ces douleurs. À la fin de sa mission, quand l'auteur a retrouvé des médecins qui avaient été affectés à d'autres zones géographiques, ceux-ci se sont montrés étonnés d’apprendre l'amélioration de ces symptômes : la plupart des femmes qu'ils avaient rencontrées dans d'autres zones faisaient état de douleurs similaires dans le bas-ventre et le bas du dos, symptômes auxquels ils n'avaient trouvé aucune solution.

\section{Efficience et efficacité du traitement en groupe}

Nous avons mené quatre groupes de thérapie différents et offert seulement deux séances en raison des contraintes de temps de la mission (3 semaines) et $\mathrm{du}$ fait que ces femmes devaient quitter leur village et leur famille pour se rendre au site de traitement le plus proche, généralement situé à une journée de marche, aucun transport public n'étant disponible. Étant donné l'extrême gravité des traumatismes dont ces femmes avaient été victimes, y compris le fait que certains villages avaient été complètement incendiés, que de très nombreuses personnes, parmi lesquelles des enfants, avaient été tuées, que de nombreux survivants avaient été torturés et mutilés, en plus de leur propre vécu d'agression sexuelle, nous pouvons faire l'hypothèse que deux séances de groupe seulement n'ont pas suffi à réduire les résultats SUD en dessous de 4.8 au post-traitement. Peut-être trois à quatre séances auraient-elles permis de réduire ces résultats de manière plus importante. Cette hypothèse est corroborée par les notes SUD des six femmes en thérapie de groupe, qui n'ont pas diminué en-deçà de 6 après deux séances et auxquelles on a offert une séance individuelle d'EMDR : à la fin de cette séance additionnelle, la moyenne de leurs notes SUD était en effet de 2.83 (voir tableau 1).

La moyenne des notes IES en post-traitement était significativement inférieure à celle des résultats en prétraitement (33 comparé à 51). Cependant, la moyenne des résultats en post-traitement était encore proche du seuil diagnostique IES de 35 (Neal et coll., 1994). Il n'est pas impossible que la note IES post-traitement de ces femmes ait été influencée négativement par une cérémonie pour le $10^{\mathrm{e}}$ anniversaire d'un massacre dans le village, qui s'est tenue la veille de l'évaluation IES. Néanmoins, ces résultats élevés suggéraient également que plusieurs femmes souffraient encore de symptômes importants et auraient eu besoin de soins supplémentaires. Il est impossible de déterminer si ces symptômes étaient liés aux agressions sexuelles ou à d'autres traumas dont ces femmes avaient été victimes. D’autres recherches pourraient donc permettre de déterminer le nombre de séances nécessaires pour entraîner une baisse significative des résultats SUD.

Un autre facteur à prendre en considération dans l'analyse des données de groupe est la mesure de la note SUD. Bien que sa validité et la pertinence de son utilisation dans la thérapie EMDR aient été confirmées par Kim et coll. (2008), ainsi que nous l'avons mentionné plus haut, certaines de ces femmes ne comprenaient pas les chiffres et nous avons utilisé une description verbale. Il est donc possible que les notes SUD donnés par les femmes du traitement en groupe aient manqué de précision. Lors des séances individuelles, le thérapeute a peut-être été plus à l'écoute et plus à même d'expliquer le résultat SUD. Il serait recommandé de standardiser les mesures des notes SUD en utilisant une description picturale comme celle qu'on utilise dans les hôpitaux pour mesurer l'intensité de la douleur des patients.

Trois femmes du traitement en groupe dont les notes SUD finales restaient élevées ont refusé la thérapie individuelle. Leur cas est à prendre en considération dans la mesure où il pourrait nous aider à mieux choisir les candidates à adresser en traitement EMDR de groupe et celles à orienter plutôt vers des séances individuelles. La première de ces trois femmes avait été abandonnée par son mari à la suite du viol. C’est là un phénomène connu qui est pris généralement en charge par les églises. Ces femmes-là vivent ainsi un double traumatisme et se retrouvent souvent dans la pauvreté et l'isolement. Pour ce genre de situation, l'auteur pense qu'il serait préférable de résoudre les problèmes sociaux et financiers de ces femmes avant de leur proposer une thérapie. La seconde femme souffrait de problèmes médicaux graves et permanents consécutifs à son agression sexuelle. L'auteur l'a adressée à l'hôpital local afin qu'elle y soit traitée. Là aussi, les problèmes médicaux doivent être pris en charge avant la mise en place d'une aide psychologique. La troisième femme avait été victime d'un viol très brutal, quatre de ses enfants avaient été tués, son mari battu et laissé handicapé et 47 membres de sa famille élargie, dont ses parents, avaient été assassinés. Ses notes SUD en début et en fin de traitement étaient de 10. Un meilleur filtrage permettrait sans doute de ne pas inclure au traitement de groupe des cas d'une telle gravité.

Il convient également de noter que 20 femmes sur 29 ont bien répondu au traitement en groupe, 9 d'entre elles rapportant des notes SUD inférieures à 3 . Nous pensons donc qu'avec un meilleur filtrage et 
plus de séances, la thérapie EMDR de groupe utilisant l'EMDR-PITG peut être un instrument thérapeutique efficace et fiable dans le traitement des personnes adultes victimes de traumas.

Bien qu'il n'en soit pas fait mention dans cet article, l'auteur a également mené une séance de thérapie de groupe avec deux groupes d'hommes victimes de traumas, qui s'est révélée tout aussi efficace. Les hommes ont bien répondu au protocole, y compris au dessin et à la technique du papillon. Nous recommandons donc que d'autres recherches soient menées sur l'EMDR-PITG, car il semble que ce soit un outil efficace pour le traitement de groupes de personnes ayant vécu les mêmes types de traumas.

\section{Limites}

Les résultats de cette étude doivent être interprétés avec beaucoup de prudence parce que nous étions limités par le temps et par les difficultés inhérentes au site lui-même. Ce n'était pas une étude contrôlée. Nous avions planifié une mission de suivi qui nous aurait permis de recueillir d'autres mesures IES posttraitement et d'approfondir l'étude de l'EMDR-PITG comme outil de traitement de l'ESPT ; malheureusement, cette mission de suivi n'a pas eu lieu.

\section{Suggestions pour de futures recherches}

Ainsi que nous l'avons mentionné plus haut, certaines des femmes de l'étude n'étaient pas aptes à participer à la thérapie de groupe. D’autres recherches portant sur les critères de sélection des participants seraient nécessaires afin de déterminer les personnes susceptibles de suivre une thérapie de groupe et celles à orienter vers une thérapie individuelle.

En République démocratique du Congo, seulement $10 \%$ des femmes et $50 \%$ des hommes sont allés à l'école. Comment rendre le protocole plus accessible aux individus qui n'ont pas cette éducation scolaire et qui rencontrent des difficultés pour s'exprimer par écrit ou par le biais de dessins?

Étant donné que le nombre de professionnels de santé mentale formés est très insuffisant dans de nombreux pays en voie de développement, pourrait-on envisager de former du personnel paraprofessionnel à la thérapie EMDR en groupe ?

Les améliorations survenues au sein de la thérapie de groupe étaient-elles spécifiques à l'EMDR ? Des recherches pourraient comparer l'EMDR à l'écoute active, qui reste le traitement recommandé et utilisé au Congo, et/ou aux interventions de soutien de groupe.
Quel est le nombre idéal de participants en thérapie EMDR de groupe ? La taille du groupe a-t-elle une influence sur les résultats du traitement?

Existe-t-il une corrélation positive entre les mesures de détresse somatique et les notes SUD ?

\section{Références}

Artigas, L., Jarero, I., Mauer, M., Lopez Cano, T., \& Alcala, N. (2000, septembre). EMDR and traumatic stress after natural disasters: Integrative treatment protocol and the butterfly hug. Poster presented at the EMDRIA Conference, Toronto, Ontario, Canada.

Bisson, J., \& Andrew, M. (2009). Psychological treatment of post-traumatic stress disorder (PTSD). Cochrane Database of Systematic Reviews, (3), CD003388.

Brewin, C. R. (2005). Systematic review of screening instruments for adults at risk of PTSD. Journal of Traumatic Stress, 18(1), 53-62.

Horowitz, M., Wilner, N., \& Alvarez, W. (1979). Impact of event scale: A measure of subjective stress. Psychosomatic Medicine, 41, 209-218.

Jarero, I., \& Artigas. L. (2010). The EMDR integrative group treatment protocol: Application with adults during ongoing geopolitical crisis. Journal of EMDR Practice and Research, 4(4), 148-155.

Jarero, I., Artigas, L., \& Hartung, J. (2006). EMDR integrative group treatment protocol: A post-disaster trauma intervention for children and adults. Traumatology, 12(2), 121-129.

Jarero, I., Artigas, L., \& Montero, M. (2008). The EMDR integrative group treatment protocol: Application with child victims of mass disaster. Journal of EMDR Practice and Research, 2(2), 97-105.

Jarero, I., Artigas, L., Uribe, S., \& Miranda, A. (2014). EMDR therapy humanitarian trauma recovery interventions in Latin America and the Caribbean. Journal of EMDR Practice and Research, 8(4), 260-268.

Kim, E., Bae, H., \& Park, Y. C. (2008). Validity of the subjective units of disturbance scale (SUDS) in EMDR. Journal of EMDR Practice and Research, 1(2), 67-72.

Ministère de la santé publique, programme national de santé mentale. (2012, décembre). Psychosocial and mental health support of the survivors of sexual violence: Standardized national module for the training of care providers in the Democratic Republic of the Congo. République démocratique du Congo.

Neal, L. A., Busuttil, W., Rollins, J., Herepath, R., Strike, P., \& Turnbull, G. (1994). Convergent validity of measures of post-traumatic stress disorder in a mixed military and civilian population. Journal of Traumatic Stress, 7, 447-455.

Organisation mondiale de la santé. (2011). Mental health atlas 2011. Genève, Suisse: Auteur.

Rothbaum, B. O. (1997). A controlled study of eye movement desensitization and reprocessing in the treatment of posttraumatic stress disordered sexual assault victims. Bulletin of the Menninger Clinic, 61(3), 317-334. 
Rothbaum, B. O., Astin, M. C., \& Marsteller, F. (2005). Prolonged exposure versus eye movement desensitization and reprocessing (EMDR) for PTSD rape victims. Journal of Traumatic Stress, 18(6), 607-616.

Shapiro, F. (1989). Efficacy of the eye movement desensitization procedure in the treatment of traumatic memories. Journal of Traumatic Stress, 2, 199-223.

Shapiro, F., \& Forrest, M. S. (1997). EMDR: The breakthrough therapy for overcoming anxiety, stress, and trauma. New York, NY: Basic Books.

Steiner, B., Benner, M. T., Sondorp, E., Schmitz, K. P., Mesmer, U., \& Rosenberger, S. (2009). Sexual violence in the protracted conflict of DRC programming for rape survivors in South Kivu. Conflict and Health, 3, 3.

Van Rood, Y. R., \& De Roos, C. (2009). EMDR in the treatment of medically unexplained symptoms: A systematic review. Journal of EMDR Practice and Research, 3, 248-263.

Wolpe, J. (1969). The practice of behavior therapy. New York, NY: Pergamon Press.

Merci d'adresser toute correspondance concernant cet article à Michel Allon, Gan Or 27, Hoshaya, Israel 17915. E-mail :michelallon@yahoo.ca 


\section{Appendice}

Étapes du protocole de traitement intégratif en groupe

Le protocole comprenait les étapes suivantes :

1. Prenez une feuille de papier A4, partagez-la en quatre et disposez-la horizontalement.

\begin{tabular}{|l|l|}
\hline & \\
\hline & \\
\hline
\end{tabular}

2. Lieu sûr : dans le rectangle supérieur gauche, faites dessiner à chaque participants une chose ou un lieu qui représentent pour lui un souvenir ou un sentiment positifs.

3. Apprenez aux participants à faire le papillon (Artigas et coll., 2000) qui tiendra lieu de stimulation bilatérale au cours de la thérapie.

4. Demandez aux participants de regarder le dessin de leur lieu sûr et faites-leur effectuer ensuite le papillon tout en fermant les yeux. Tout au long du protocole, à chaque fois, la durée de la stimulation doit avoisiner 1 minute.

5. Demandez à chaque participant d'expliquer au groupe ce que représente son lieu sûr. De cette manière, chaque participant contribue au sentiment de relaxation et de sécurité du groupe.

6. Répétez l'étape 4, où chaque participant pratique le papillon tout en imaginant son lieu sûr.

7. Dans le coin supérieur droit de la feuille, demandez aux participants de dessiner une image qui représente le trauma qu'ils traiteront ensuite.

8. À côté du dessin, faites-leur écrire un mot qui représente l'émotion qu'ils ressentent concernant le dessin de leur trauma.

9. Dans un petit cercle, sur cette case, faites-leur écrire leur SUD (0-10) décrivant l'intensité de cette émotion.

10. Demandez-leur de fermer les yeux et de visualiser le dessin du trauma tout en faisant le papillon.
11. À ce stade, ne demandez pas aux participants de partager les dessins de leur trauma avec le groupe, car cela pourrait déclencher trop d'activation traumatique au sein du groupe.

12. Demandez ensuite aux participants de dessiner, dans le cadre inférieur gauche de la feuille, une image de ce qui s'est présenté à eux lorsqu'ils traitaient l'image du trauma en faisant le papillon.

13. Faites-leur ensuite écrire l'émotion qui monte avec ce nouveau dessin, et notez le résultat SUD $(0-10)$ associé à cette émotion.

14. Les yeux fermés, demandez-leur d'imaginer le nouveau dessin tout en faisant le papillon.

15. Demandez ensuite aux participantes de dessiner, dans le rectangle inférieur droit de la feuille, une image de ce qui leur est venu pendant qu'ils traitaient leur dernier dessin (dans le coin inférieur gauche) avec le papillon.

16. Faites-leur ensuite écrire l'émotion qu'ils ressentent maintenant, avec ce nouveau dessin, puis inscrivez le SUD (0-10) associé à cette émotion.

17. Les yeux fermés, demandez-leur d'imaginer le nouveau dessin tout en faisant le papillon.

18. Ensuite, demandez aux participantes de revenir au dessin du trauma et réévaluer l'émotion associée à l'aide de l'échelle SUD (0-10). En bas à droite de la page, demandez-leur d'indiquez et d'encerclez le résultat SUD.

19. Demandez maintenant aux participants de parler entre eux du retraitement de leur trauma. Notez le nom de ceux dont le résultat est encore élevé à la fin du retraitement. Déterminez s’il est nécessaire de poursuivre le retraitement immédiatement ou s'ils peuvent attendre la prochaine séance de groupe.

20. Pour terminer la séance, demandez aux participants de former un cercle, chacun faisant face au dos de la personne devant lui. Pendant qu'ils imaginent leur lieu sûr, demandez-leur de tapoter bilatéralement les épaules de la personne devant eux. 